\title{
A fusion optimization algorithm of network element layout for indoor positioning
}

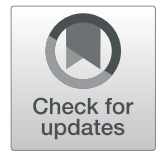

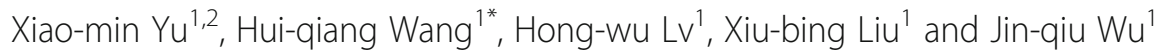

\begin{abstract}
The indoor scene has the characteristics of complexity and Non-Line of Sight (NLOS). Therefore, in the application of cellular network positioning, the layout of the base station has a significant influence on the positioning accuracy. In three-dimensional indoor positioning, the layout of the base station only focuses on the network capacity and the quality of positioning signal. At present, the influence of the coverage and positioning accuracy has not been considered. Therefore, a network element layout optimization algorithm based on improved Adaptive Simulated Annealing and Genetic Algorithm (ASA-GA) is proposed in this paper. Firstly, a three-dimensional positioning signal coverage model and a base station layout model are established. Then, the ASA-GA algorithm is proposed for optimizing the base station layout scheme. Experimental results show that the proposed ASA-GA algorithm has a faster convergence speed, which is 16.7\% higher than the AG-AC (Adaptive Genetic Combining Ant Colony) algorithm. It takes about 25 generations to achieve full coverage. At the same time, the proposed algorithm has better coverage capability. After optimization of the layout of the network element, the effective coverage rate is increased from 89.77 to $100 \%$ and the average location error decreased from 2.874 to $0.983 \mathrm{~m}$, which is about 16\% lower than the AG-AC algorithm and 22\% lower than the AGA (Adaptive Genetic Algorithm) algorithm.
\end{abstract}

Keywords: Non-Line of Sight (NLOS), K-Coverage, Fusion algorithm, Base station layout, Convergence rate

\section{Introduction}

Statistics show that about $80 \%$ of people's living and working environment is indoors. The location service in the indoor environment plays an extremely important role in the control of objects in industrial production lines, location and navigation in public places, the care of young and old people, and intelligent entertainment $[1,2]$. In order to improve the effective coverage of the indoor positioning signal and the indoor positioning accuracy, a reasonable base station layout is particularly important, which can improve the positioning accuracy while reducing the deployment cost.

Due to the NLOS of the indoor environment and the complexity of the indoor structure, the Global Positioning System (GPS) is not available for indoor positioning. Because indoor environment is complex, all sorts of electromagnetic wave can produce the change of signal characteristic because of reflex, refraction, and diffraction.

\footnotetext{
* Correspondence: 52410660@qq.com

${ }^{1}$ College of Computer Science and Technology, Harbin Engineering

University, Harbin, Heilongjiang province, China

Full list of author information is available at the end of the article
}

We can use this kind of characteristic change to realize indoor communication, indoor position, etc. Electromagnetic (EM) waves with helical wave front carry orbital angular momentum (OAM), which is associated with the azimuthal phase of the complex electric field. OAM is a new degree of freedom in EM waves and is promising for channel multiplexing in the communication system [3, 4]. Currently, positioning technologies based on Bluetooth, WiFi [5], and UWB [6] can achieve good positioning effects, but lack a unified wide-area positioning network. In the coming $5 \mathrm{G}$ era, the integration of communication and navigation networks of heterogeneous cellular networks is an important trend in indoor positioning $[7,8]$, which can provide communication and positioning services at the same time avoiding additional resource overhead.

However, the complex of the indoor environment structure leads to signal loss, reflection, refraction, and diffraction and even the positioning terminal cannot be covered by multiple base stations at the same time, resulting in an increasement in positioning error or failure to provide positioning requirements. In the 3D 
positioning, the positioning terminal must ensure that the coverage of at least four base stations is received at the same time to satisfy the positioning condition. By properly arranging the layout of the base station, the number of the first path received by the terminal can be effectively improved, thereby improving the positioning accuracy.

The main contributions of this paper are:

- Presents an indoor positioning base station layout optimization method based on ASA-GA, which taking the two factors-positioning signal coverage ratio and positioning accuracy-into consideration to optimize the base station layout scheme.

- Takes the improved adaptive genetic algorithm as the main body of the algorithm, and integrates the improved simulated annealing mechanism to further adjust and optimize the population, so can improve the convergence speed and optimization quality of the algorithm.

The rest of this paper is organized as follows. Related work is presented in Section 2. Section 3 describes network element optimization layout model and ASA-GA algorithm. In Section 4, the simulation scenes are described. In Section 5, the performance evaluation of the ASA-GA algorithms in terms of signal coverage, positioning error, and iteration number is given. Finally, Section 6 gives conclusions and outlines the future work.

\section{Related works}

The positioning accuracy can be improved from two aspects. The first one is to place the base station so that each point of the positioning area is covered by at least 4 base stations at the same time. The second is to reduce the GDoP (Geometric Dilution of Precision), thereby reducing the average positioning error of the space [9]. The location selection of base station in space is always regarded as NP-hard problem [10]. Finding the best base station layout scheme is still challenging, even if the search space is roughly represented, the enumeration search is invalid [9]. Therefore, this type of problem only solves approximate or suboptimal solutions [11]. Heuristic algorithm can improve the search speed [12]. The existing base station layout algorithm can be divided into two base station layout optimization methods based on random geometry and heuristic search.

In terms of random geometry, Bais et al. [9] laid out indoor base stations in a square shape, which solved the problem of signal coverage and improved the positioning error. However, the irregularity of buildings makes all base stations have a square layout difficulty. In literatures
[13-16], many existing methods consider the localization performance of one or several specific points. Andrews et al. [17] model the layout of a cellular network using a homogeneous Poisson point process. The scenario modeling of the base station location in the cellular network means that the deployed base stations are completely independent of each other. The work of Zhou et al. [18] is an extension of these methods. They studied placing four base stations in a rectangular area, and research on positioning performance and effect. A solution based on Monte Carlo simulation is proposed for the difficulty of problem analysis. It is also confirmed by Chen et al. [19] that the optimal placement of the four base stations is rectangular.

Base station layout intelligent algorithm based on heuristic search is more adaptable and easily to model [20]. Zhang et al. [21] proposed a solution based on Simulated Annealing (SA) algorithm, but the initial value of "temperature" and the rate of decline in the simulated annealing algorithm need to be repeated several times to determine. Pereira et al. [22] used the particle swarm optimization algorithm based on the idea of group intelligent optimization to apply to the base station optimization problem, which is easy to modify the objective function, and can be implemented in parallel with good scalability. However, because the population loses more diversity information in the search space, it is easy to fall into the local optimal solution. Meng et al. [23] proposed the introduction of the Pareto optimal domain based on the traditional genetic algorithm (GA) layout scheme, and proposed a high-performance NSGA-II algorithm. This algorithm is a heuristic search algorithm and is also easily rewritten as a parallel processing version.

The single algorithm has its own performance defects, which leads to unsatisfactory optimization results. Therefore, domestic and foreign scholars put forward the improved fusion optimization algorithm strategy. Literature [24] also proposed the base station optimization scheme based on Genetic Algorithm. However, all of them have the characteristics of weak global search ability and easy to fall into the local optimal solution. Wang et al. [25] optimized the base station layout using adaptive genetic algorithm and ant colony algorithm (AG-AC). Firstly, the cross and mutation probabilities in the traditional genetic algorithm are adjusted to make them constantly change with the iteration of the algorithm, so can achieve the purpose of self-adaptation and generate the primary network element layout. Then, adaptive ant colony algorithm is applied on the primary network element layout to change the pheromone of traditional ant colony algorithm into a variable that changes constantly with the iteration of the algorithm, so as to reduce the risk of ant colony algorithm falling 
into local optimal solution and then generate the final network element layout. The average error after two steps' optimization is significantly improved compared with that before fusion. But this approach is simply a splicing of two algorithms, and the convergence speed of the algorithm is not significantly improved. Gharghan et al. [26] proposed hybrid Particle Swarm Optimization-Artificial Neural Network (PSO-ANN). This algorithm adopts the feedforward neural network model and uses the Levenberg-marquardt training algorithm to estimate the distance between the moving node and the anchor node. Although the positioning accuracy is improved, the training of feedforward neural network needs a lot of samples; otherwise, it cannot converge to the global minimum or the local minimum with good enough. In terms of continuous optimization, Ying Gao et al. [27] first introduced the idea of annealing particle swarm optimization. This algorithm combines the advantages of PSO algorithm, such as global optimization ability, fast calculation speed and simple implementation, and the simulated annealing algorithm's ability to jump out of local optimal solution. It avoids the disadvantage of PSO falling into local extremum and improves the convergence speed of PSO at the later stage of evolution. Zhang et al. [28] proposed a hybrid simulated annealing genetic optimization algorithm in order to improve the convergence speed of the genetic algorithm. In the early stage, the standard genetic algorithm was adopted for optimization, and the optimized results of the genetic algorithm were annealed. Although the algorithm improved the positioning accuracy, it cannot converge to the extreme point in the later stage, which makes the algorithm unstable.

\section{Method}

According to the needs of positioning, this paper optimizes the location of multiple network elements in space to ensure the coverage of positioning signals and improve the positioning stability and accuracy of terminals. Firstly, the optimization model of the network element is established, and the optimization problem of the network element layout is transformed into simple discrete optimization problem. Then, according to the model and the disadvantages and advantages of the single algorithm, an improved adaptive genetic annealing fusion optimization algorithm is proposed. This algorithm takes the improved AGA as the main body of the algorithm, and integrates the improved simulated annealing mechanism to further adjust and optimize the population, so can improve the convergence speed and optimization quality of the algorithm.

\subsection{Network element optimization layout model}

Signal coverage in indoor location is an important indicator. In the three-dimensional (3D) indoor positioning, the to-be-positioned point receives at least four network element transmission signals, which can be regarded as effective coverage. The $3 \mathrm{D}$ indoor space is modeled using the probing model and K-coverage [11, 29], and the spatial positioning signal coverage is finally calculated. The Euclidean distance is used to solve the positioning error of each terminal, and the minimum positioning error is taken as the objective function of optimization.

\subsubsection{D coverage rate model}

\section{- Detecting Model}

There are $N$ network elements, and the coordinates of the $i$ th network element are set to $A_{i}\left(x_{i}, y_{i}, z_{i}\right), i=(1,2$, $\ldots, N)$. Set the detection radius of the network element $A_{i}\left(x_{i}, y_{i}, z_{i}\right)$ as $r_{i}$. Then, the detection area of the network element is the spherical area with the radius $r_{i}$, where the location of the network element $A_{i}$ is located at ( $x_{i}$, $\left.y_{i}, z_{i}\right)$.

$$
V_{i}:\left(x-x_{i}\right)^{2}+\left(y-y_{i}\right)^{2}+\left(z-z_{i}\right)^{2} \leq r_{i}^{2}
$$

$V_{i}$ is the coverage detection region of the network element $A_{i}$, that is, the effective region is denoted as $V e_{i}$. The region other than $V_{i}$ is the undetectable region of the network element $A_{i}$. Set the target 3D space region as $V$, then the effective region $V e_{i}=V_{i} \cap V$.

Let network element layout is $S$, then $S=\left(A_{1}, A_{2}, A_{3}\right.$, $\left.\ldots, A_{N}\right)$. The detection area of each network element is $V_{i}, i=(1,2, \ldots, N)$, and the total detection area of $N$ network elements is $\cup_{i=1}^{N} V_{i}$. The effective detection area of each network element is set to $V e_{i}, i=(1,2, \ldots, N)$, then $V e_{i}=V_{i} \cap V$. Set the effective total detection region of $N$ network element as $\cup_{i=1}^{N} V e_{i}$.

\section{- K-coverage method}

The ranging-based 3D positioning algorithm receives at least four network element signals at the same time. In this case, K-coverage effective positioning point is used, that is, $K \geq 4$ is the effective coverage; otherwise, there is coverage vulnerability. For the irregularities of complex and diverse indoor space shapes, this paper uses the cube segmentation method to segment the location region.

Set the indoor positioning space area as $V$ and the side length of the cube is $l$.The region $V$ is divided into $M$ small cube regions, i.e., $M=\frac{V}{l^{3}}$. Set the body centered coordinates of each small cube region as $B_{j}\left(x_{j}, y_{j}, z_{j}\right)$, 
$j=(1,2, \ldots, M)$, and replace the small cube area with the cube center. Therefore, the coverage of the network element for each small cube can be approximated as the coverage of the cube center $B_{j}\left(x_{j}, y_{j}, z_{j}\right) . S$ is used for a network element layout, $S=\left(A_{1}, A_{2}, A_{3}, \ldots, A_{N}\right)$ and the network element coordinates are $A_{i}\left(x_{i}, y_{i}, z_{i}\right), i=(1,2, \ldots$, $N)$. When $\left(x_{i}-x_{j}\right)^{2}+\left(y_{i}-y_{j}\right)^{2}+\left(z_{i}-z_{j}\right)^{2} \leq r_{i}^{2}, B_{j}$ is considered to be covered by network element $A_{i}$. Let the variable $K_{i j}$ denote the case where $B_{j}$ is covered by the network element $A_{i}$, where $j=(1,2, \ldots, M), i=(1,2, \ldots, N)$. Then, the expression $K_{i j}$ is shown in (2):

$$
K_{i j}= \begin{cases}1 & \left(x_{i}-x_{j}\right)^{2}+\left(y_{i}-y_{j}\right)^{2}+\left(z_{i}-z_{j}\right)^{2} \leq r_{i}{ }^{2} \\ 0 & \left(x_{i}-x_{j}\right)^{2}+\left(y_{i}-y_{j}\right)^{2}+\left(z_{i}-z_{j}\right)^{2}>r_{i}{ }^{2}\end{cases}
$$

According to the definition of K-coverage, the coverage number of the target node $B_{j}$ is $K_{j}$, and the target node $B_{j}$ is overwritten by the network element $K_{j}$. The expression of $K_{j}$ is as shown in Formula (3):

$$
K_{j}=\sum_{i=1}^{M} K_{i j}
$$

In indoor positioning, each node to be located should be covered by at least four network elements. Let, the variable $E_{j}$ indicate whether point $B_{j}$ is effectively covered. While the value of $E_{j}$ is equal to 1 , that means the point $B_{j}$ is effectively covered, vice versa. The expression of $E_{j}$ is shown in (4):

$$
E_{j}=\left\{\begin{array}{lc}
1 & K_{j} \geq 4 \\
0 & K_{j}<4
\end{array}\right.
$$

The area coverage expression under the network element layout $S$ is shown in formula (5):

$$
f_{c}(S)=\frac{\sum_{j=1}^{n^{3}} E_{j}}{n^{3}}
$$

\subsubsection{Network element layout}

In the network element layout process, $A_{i}\left(x_{i}, y_{i}, z_{i}\right)$ is used for the coordinates of the $i$ th network element, where $i=1,2, \ldots, N$. M nodes are collected to represent the users in the indoor environment, the coordinate of the $j$ th user is $B_{j}\left(x_{j}, y_{j}, z_{j}\right)$ and the measurement coordinate of the $j$ th user is $B_{j}{ }^{\wedge}\left(\hat{x}_{j}, \hat{y}_{j}, \hat{z}_{j}\right)$. It is assumed that the positioning probability of each positioning node is the same. Define $S^{\prime \prime}, P$, and $\hat{P}$ as $\left(A_{1}, A_{2}, A_{3}, \ldots, A_{N}\right),\left(B_{1}, B_{2}, \ldots\right.$, $\left.B_{M}\right)$, and $\left(\hat{B}_{1}, \hat{B}_{2}, \ldots, \hat{B}_{M}\right) . S$ represents the layout scheme of $N$ network elements, $P$ denotes the position of all points of $M$ users in the indoor environment while $\hat{P}$ is the measurement position.

In the case of network element layout $S$, the average positioning error of $M$ users in the indoor environment is:

$$
f(S)=\frac{1}{M} \sum_{j=1}^{M} \operatorname{Erro}_{j}(S)
$$

$f(S)$ is used for the average positioning error. In the indoor positioning, it is determined whether each positioning point receives signals of at least 4 network elements at the same time by determining whether the value of $E$ that corresponds to each user is 1 . When the user's $E$ value is 1 , the positioning accuracy is maximized, where $\operatorname{Erro}_{j}(S)$ is used for the positioning error of the $j$ th user [22].

By measuring the distance between the network element and the user, the positioning accuracy is calculated, and the layout of the network element is evaluated, finally obtain the optimized layout results.

\subsection{ASA-GA algorithm design \\ 3.2.1 Design of the adaptive simulated annealing (ASA) algorithm}

Simulated annealing algorithm is a common heuristic algorithm whose performance largely depends on its components and parameters. It mainly includes the methods of generating new states, the design of cooling control function, and the termination conditions of the algorithm [30]. The traditional simulated annealing algorithm is used in the open-loop control mode, so the neighborhood search results have no feedback effect on the annealing process. This paper presents a fast adaptive simulated annealing algorithm, which adopts closedloop feedback control to combine the neighborhood search and temperature control by selecting appropriate methods and algorithm termination conditions for generating new states. The algorithm can dynamically determine the temperature parameters and the changes in the number of different neighborhood searches. The specific process after improvement is as follows:

In space $V$, the base station layout $S$ represents a feasible solution, and the energy function is the average error of the positioning, also called the objective function of the algorithm optimization. As shown in Eq. (6), the minimization of the objective function is the optimal solution of the layout. As for the cooling progress function, this paper uses the exponential cooling strategy to control, expressed as: 


$$
T(k)=T_{0} \alpha^{k^{1 / 2}}
$$

where $T_{0}$ is the initial temperature, and $k$ is the temperature drop coefficient.

Let $P$ be the state transition probability, indicating the probability of going from one base station $S$ to another base station layout $S^{\prime}$, related to the current temperature parameter $T_{i}$, where $T_{i}$ representing the temperature of the $i$ th iteration, expressed as:

$$
P= \begin{cases}1, & f\left(S^{\prime}\right) \leq f(S) \\ \exp \left[f(S)-f\left(S^{\prime}\right) / T_{i}\right], & f\left(S^{\prime}\right)>f(S)\end{cases}
$$

Since using the simulated annealing algorithm solves the new solution in the neighborhood area of the current solution, to ensure that the individual of the new solution does not exceed the boundary, the boundary station number $q^{\prime}$ in the new solution is converted as follows:

$$
q^{\prime}= \begin{cases}q+\left(q_{\text {right }}-q\right) \delta\left(T_{i}\right) \varepsilon, & U(0,1)=0 \\ q-\left(q-q_{\text {left }}\right) \delta\left(T_{i}\right) \varepsilon, & U(0,1)=1\end{cases}
$$

where $q_{\text {left }}$ and $q_{\text {right }}$ are respectively the minimum and maximum of the base station number, $\varepsilon$ is the random number between $(0,1), U(0,1)$ is the control value of randomly selecting 0 or $1, \delta\left(T_{i}\right)$ is the disturbance quantity, which decreases with the decrease of $T_{i}$, and finally with $\delta\left(T_{i}\right) \rightarrow 0$ the algorithm converges.

\subsubsection{Design of the adaptive genetic algorithm with annealing thought}

- Fitness function

The goal of the network element optimization layout is to improve the positioning accuracy, that is, the positioning error of the point to be located is the smallest. Therefore, the population fitness function can be expressed as Eq. (6).

\section{- Selecting operation}

In the optimization of network element layout based on adaptive genetic algorithm, the selection operation is to select a good individual from the population, where the probability of individuals being selected is expressed as:

$$
P_{k}=1-\frac{f_{k}}{\sum_{i=1}^{n} f_{i}}
$$

In which, $P_{k}$ is the probability that the population individual $S_{k}$ is selected, and $f_{i}$ is the fitness function of population individual $S_{k}$.

\section{- Adaptive selection of crossover operators}

This paper makes the following adaptive improvements for crossover probabilities:

$$
p_{c}=\left\{\begin{array}{c}
k_{1} \cdot \frac{f^{\prime}-f_{\text {min }}}{f_{\text {avg }}-f_{\text {min }}}, f^{\prime} \leq f_{\text {avg }} \\
k_{2}, \quad f^{\prime}>f_{\text {avg }}
\end{array}\right.
$$

where $f_{\text {avg }}$ represents the average fitness value of all individuals in the population, and $f_{\min }$ represents the minimum fitness value of individuals in the population, that is, the minimum positioning error under the network element layout. $f$ is used for the fitness value of the current network element layout, that is, the average positioning error under the current network element layout. It can be seen from formula (11) that the smaller the value of $f_{\text {avg }}-f_{\min }$ is, the closer $f_{\text {avg }}$ is to $f_{\min }$, and the more the layout of network elements is to the optimal solution. According to the network element optimization scenario, network element layout individuals with lower fitness are assigned lower $p_{c}$, is conducive to the preservation of good individuals. On the contrary, network element layout individuals with higher fitness are assigned larger $p_{c}$. The crossover probability of individuals is not only determined by $f_{\text {avg }}-f_{\text {min }}$, the closer $f_{\text {avg }}$ is to $f_{\text {min }}$, the smaller the error caused by network element layout. As shown in algorithm 1. The variable pop represents population, pop_size represents population size, chromo represents chromosome while chromo_size represents chromosome length.

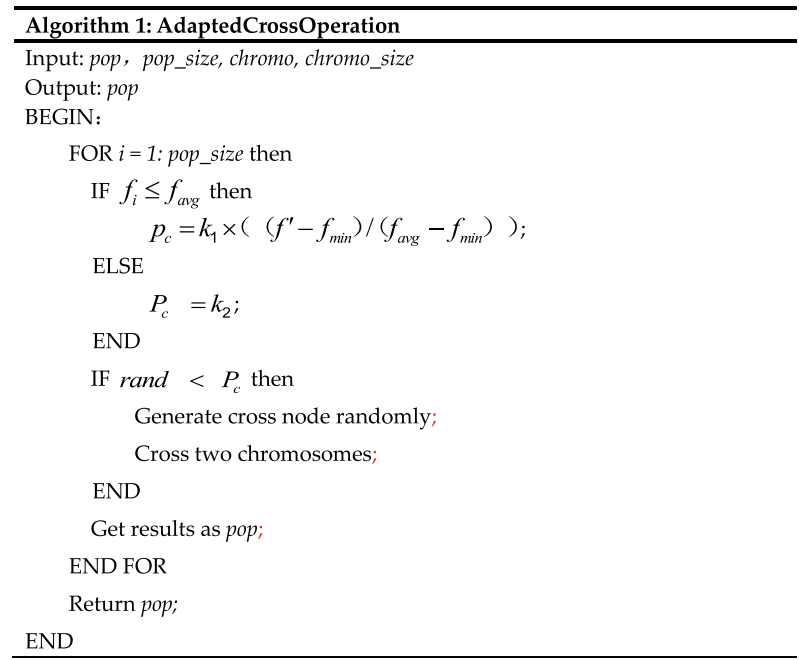


- Adaptive selection of mutation operators

According to the adaptability of the current network element layout and the average fitness of the entire population, the adaptive mutation probability is selected. If the current layout fitness is greater than the average fitness, the mutation probability is smaller. On the contrary, the mutation probability is larger. The adaptive mutation probability formula is as follows:

$$
p_{m}=\left\{\begin{array}{c}
k_{3} \frac{f^{\prime}-f_{\text {min }}}{f_{\text {avg }}-f_{\text {min }}}, f^{\prime} \leq f_{\text {avg }} \\
k_{4}, \quad f^{\prime}>f_{\text {avg }}
\end{array}\right.
$$

Similar as Formula (11), when the fitness of the individual obtained by the variation is greater than the fitness of the current individual layout, the variation result is accepted. What is different is that annealing steps are added into the adaptive mutation operation here. When the individual mutates, the same as formula (8), the mutation operation is accepted with a probability $\exp \left[\left(f^{\prime \prime}-f^{\prime}\right) \times(1 / G)\right]$, where $f^{\prime \prime}$ is used for the fitness value of the individual after the variation, that is the average positioning error. $G$ is used for the number of current generations.

\section{Algorithm 2: AdaptedMutationOperation}

Input: pop, pop_size, chromo, chromo_size

Output: pop

BEGIN:

\%Perform mutation operation, current layout $S$

FOR $i=1$ : pop_size then

IF $f_{i}<f_{\text {avg }}$ then

$$
p_{m}=k_{3} \cdot\left(f-f_{\text {min }}\right) /\left(f_{\text {arg }}-f_{\text {min }}\right) \text {; }
$$

\section{ELSE}

$$
p_{m}=k_{4} \text {; }
$$

END

IF rand $<p_{m}$ then

$\%$ Generate mutate node randomly

Mutating a chromosome $S^{\prime}$

IF $f\left(S^{\prime}\right)<f(S)$

$$
S=S^{\prime} \text {; }
$$

\section{ELSE}

According to $\exp \left[\left(f^{\prime \prime}-f^{\prime}\right)^{*}(1 / G)\right]$ accepting $S^{\prime}$;

\section{END}

\section{END}

Get results as pop;

\section{END FOR}

\section{Return pop;}


The purpose of improving the adaptive genetic algorithm is to prevent GA from falling into the local optimal solution. The algorithm uses the network element layout below the average fitness to find the optimal solution in the indoor positioning space. The network elements layout in this situation needs to be completely disrupted, so the value of $k 4$ is set as 0.5 , similarly, the value of $k 3$ is also set as 0.5 . Set $k 1=k 2=1.0$ at the same time.

\subsubsection{ASA-GA algorithm}

The genetic algorithm can find an optimal solution from the whole, but it may fall into a local optimum, which can be avoided by the simulated annealing algorithm. In view of the shortcomings of slow convergence and poor quality of single intelligent optimization algorithm, a network layout ASA-GA optimization algorithm is proposed. The algorithm firstly adaptively improves the simulated annealing algorithm and the genetic algorithm. Secondly, the adaptive genetic algorithm is used as the optimization body of the ASA-GA algorithm. In addition, annealing mechanism is added into the adaptive mutation operation. The specific steps of ASA-GA algorithm fusion are as follows:

- Set the initial value of the algorithm's parameters, including population size $n$, the number of reproductive generations $G$, crossover probability $p_{c}$ and mutation probability $p_{m}$, and the $T_{0}$ and $k$ value of simulated annealing algorithm, and set an generation number $T_{g}$.

- Calculate the fitness of the individuals in the generated population, and record the optimal individuals, select the paired individuals according to Eq. (10) Perform the adaptive crossover and mutation operations on the individuals using Eqs. (11) and (12). The fitness of each individual in the newly generated population was calculated, and the individual with high fitness value was selected for the transfer step III to conduct annealing optimization gene operation.

- Let us make the cycle counter of simulated annealing algorithm $t$, the simulated annealing operation is performed on the individuals with high fitness in the new population and optimize individual genes. Calculate the disturbance value according to (8). The probability is accepted according to the formula (9). If accepted, $t=t+1$, otherwise, $t$ remains unchanged. The individuals with the least fitness in the population were replaced with the layout results after annealing.

- If the reproductive generation number $T_{g}$ is less than $G$, then $T_{g}=T_{g}+1$, return II, otherwise, end the optimization process and output the result of base station layout.

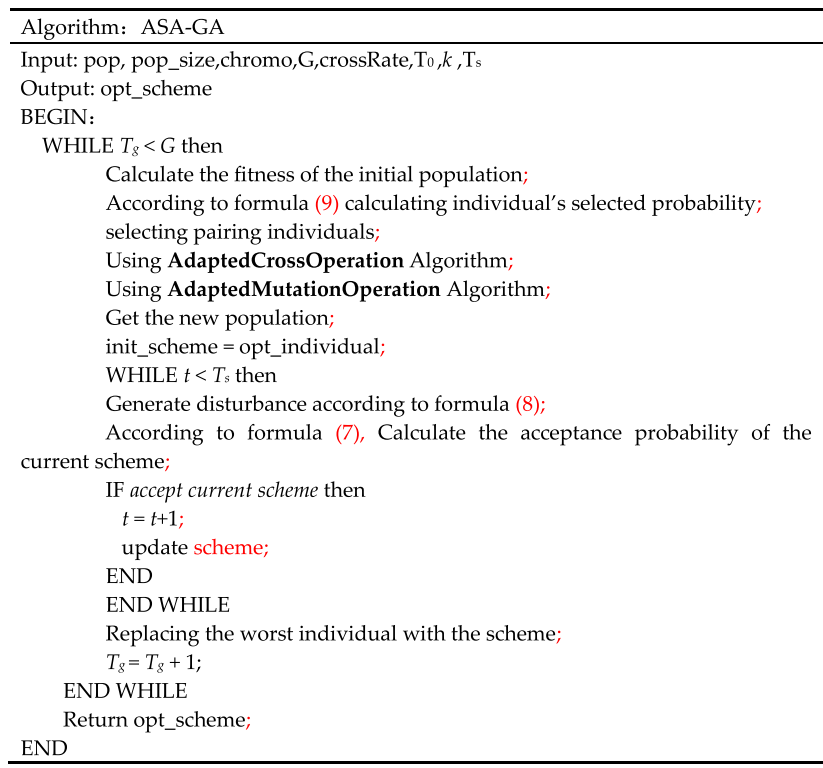

\section{Simulation experiment}

In the simulation experiment of network element layout, the experimental scene was set as a "double L" region, with a total length of $24.1 \mathrm{~m}$ and a width of $17.8 \mathrm{~m}$. According to the actual situation, for the sake of simplicity, in the layout of the network element, select $3 \mathrm{~m}$ from the ground height to divide the $1 \mathrm{~m} \times 1 \mathrm{~m}$ grid. Due to the large number of multipath generated in the deployment of ceiling elements, the positioning error is greatly reduced. Therefore, the paper select a location near the wall as the deployment of base station, a total of 102 locations for network deployment. The selected position of the terminal is $1.2 \mathrm{~m}$ away from the ground, and the $3 \mathrm{D}$ grid of $1 \mathrm{~m} \times$ $1 \mathrm{~m} \times 1 \mathrm{~m}$ is divided. Two hundred sixty-four terminals are located at the center of gravity of the grid. The test scenario is shown in Fig. 1. The yellow dot indicates the network element location to be installed and the green dot indicates the user terminal location.

Before the simulation analysis, the parameters of the ASA-GA fusion algorithm are initialized. In the experimental simulation, eight network elements are preinstalled in the positioning area. Both the simulated annealing algorithm and the ant colony algorithm are iterated for 50 times. The population of genetic algorithm is set as 50 , the genetic times are set as 50 , and the number of ants of ant colony algorithm is set as 50 . The algorithm parameter settings are shown in Table 1.

\section{Results and discussion}

In the above positioning scenario, there are 8 optimal deployment positions number of network elements, namely, 16, 29, 33, 43, 81, 88, 95, and 98. The first 


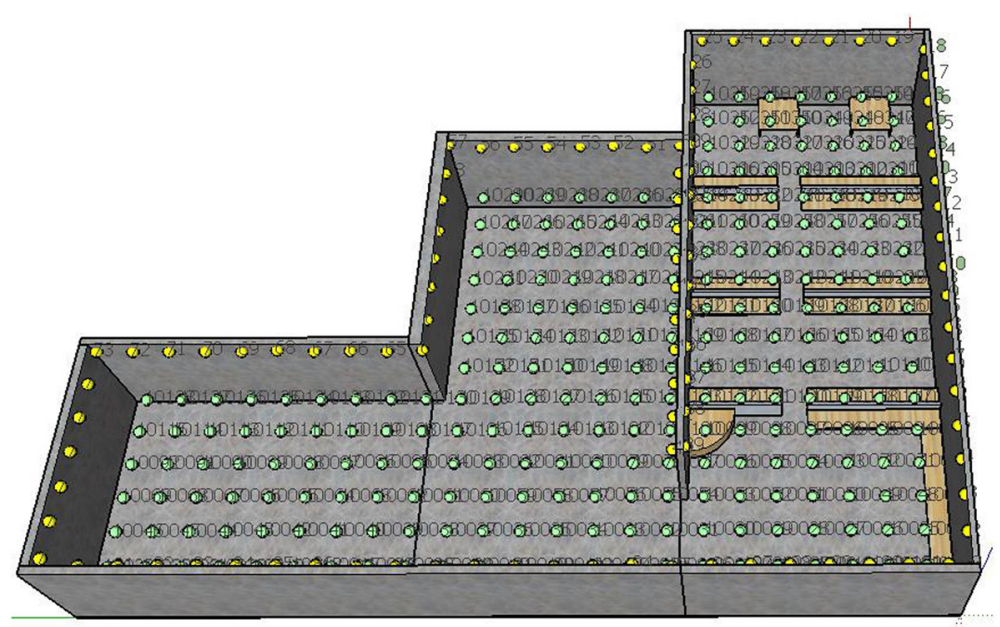

Fig. 1 Simulation optimization scenario. In the simulation experiment of network element layout, the experimental scene was set as a "double L" region, with a total length of $24.1 \mathrm{~m}$ and a width of $17.8 \mathrm{~m}$. According to the actual situation, select $3 \mathrm{~m}$ from the ground height to divide the 1 $\mathrm{m} \times 1 \mathrm{~m}$ grid. A location near the wall was selected as the deployment of base station, a total of 102 locations for network deployment. The selected position of the terminal is $1.2 \mathrm{~m}$ away from the ground, and the 3D grid of $1 \mathrm{~m} \times 1 \mathrm{~m} \times 1 \mathrm{~m}$ is divided. Two hundred sixty-four terminals are located at the center of gravity of the grid. The yellow dot indicates the network element location to be installed and the green dot indicates the user terminal location

diameter coverage of the positioning area after the layout optimization of network elements is shown in Fig. 2.

According to the coverage of the first diameter in Fig. 2, the number of the first diameter is mainly $5 \sim 7$. Compared with the positioning signal coverage before the layout optimization of the network element, the coverage rate statistical diagram is shown in Fig. 3.

It can be seen from the above figure that before the network element layout is optimized, the effective positioning signal coverage rate of that the first diameter (i.e., direct arrival signals) number is greater than or equal to 4 is about $90 \%$, and about $10 \%$ of the signal coverage holes exist. After adopting the ASA-GA fusion optimization algorithm proposed in this paper, the positioning signal coverage rate reaches $100 \%$ coverage, and the number of first diameter is mainly distributed from 5 to 7 . Through the experiment, the change of positioning accuracy before and after the layout of the network element is obtained, and the positioning accuracy is obviously improved with the increase of the coverage rate. The changes in coverage rate and positioning accuracy before and after optimization are shown in Table 2.

After the optimization of network elements layout, the effective positioning signal coverage rate increased from 89.77 to $100 \%$, the coverage rate increased by $10.23 \%$, and the average positioning error decreased from 2.874 to $0.983 \mathrm{~m}$. According to the cumulative distribution of positioning errors before and after network element layout optimization shown in Fig. 4, the number of terminals with positioning errors within $1 \mathrm{~m}$ accounts for about $68 \%$ of the total, and the number of terminals with positioning errors within $2 \mathrm{~m}$ accounts for about $85 \%$. The ASA-GA algorithm's positioning accuracy and coverage rate have been significantly improved, which proves the effectiveness of the ASA-GA hybrid optimization algorithm proposed in this paper.

Comparing the proposed ASA-GA fusion optimization algorithm with the AGA and AG-AC joint optimization algorithm proposed in [25], the cumulative error distribution of the three algorithms is shown in Fig. 5. After the optimization of ASA-GA fusion algorithm, the positioning error of target nodes within $3 \mathrm{~m}$ accounts for about $94 \%$ of the total, within $2 \mathrm{~m}$ accounts for about

Table 1 Algorithm parameter settings

\begin{tabular}{lllll}
\hline & Population quantity & Ant quantity & Genetic times & Iteration times \\
\hline ASA-GA & 50 & --- & 50 & 50 \\
AG-AC & 50 & 50 & 50 & 50 \\
AGA & 50 & --- & 50 & -- \\
\hline
\end{tabular}




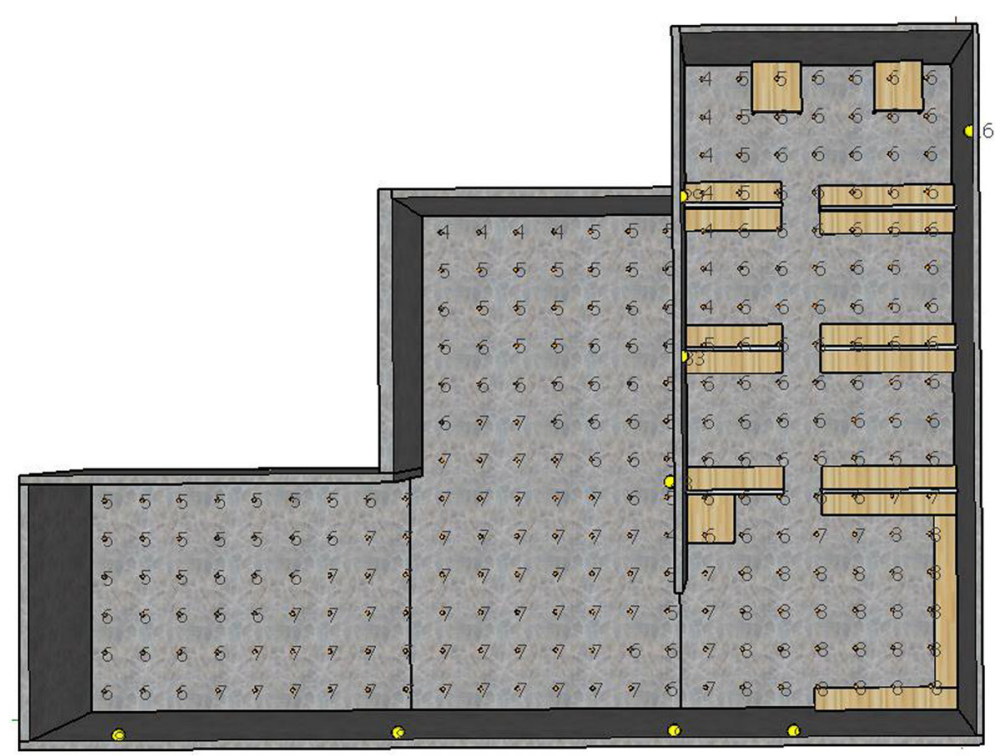

Fig. 2 The first diameter coverage. In the above positioning scenario, there are 8 optimal deployment positions number of network elements, namely, $16,29,33,43,81,88,95,98$. The number of the first diameter that the user terminal received is represented by the digit of corresponding location

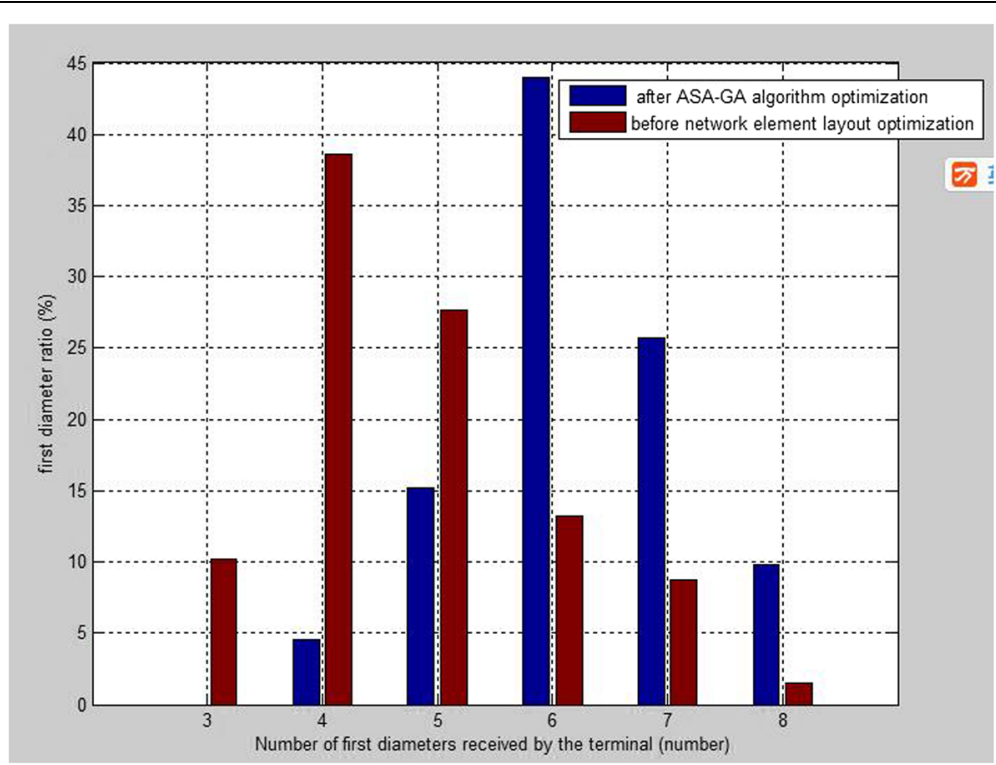

Fig. 3 The first diameter coverage statistics chart. The brown columns represent the positioning signal coverage before the layout optimization of the network element. The blue columns represent the positioning signal coverage after the layout optimization of the network element 
Table 2 Changes before and after optimization of network element layout

\begin{tabular}{lll}
\hline & Before network element layout optimization & After ASA-GA algorithm optimization \\
\hline Average positioning error & $2.874 \mathrm{~m}$ & $0.983 \mathrm{~m}$ \\
Location signal coverage rate & $89.77 \%$ & $100 \%$ \\
\hline
\end{tabular}

$85 \%$, and within $1 \mathrm{~m}$ accounts for about $68 \%$, which is about $16 \%$ better than that of AG-AC algorithm and $22 \%$ better than AGA algorithm.

The variation of positioning signal coverage during the optimization of the three algorithms is shown in Fig. 6 . Experimental results show that ASA-GA fusion algorithm needs to be inherited for 25 generations, AG-AC algorithm needs to be inherited for 30 generations, and AGA needs to be propagated for 38 generations to achieve full coverage.

\section{Conclusion}

Due to the complexity and the characteristics of nonline-of-sight of indoor scene, in the application of cellular network positioning, the influence of indoor three-dimensional positioning signal coverage and positioning accuracy has not been considered in the base station deployment. This paper proposes an adaptive genetic algorithm based on fusion annealing indoor positioning base station layout method. This algorithm adopts an adaptive way to control crossover and mutation probability, avoids sharp rise and fall, and makes the crossover and mutation operations tend to be stable, thus ensuring the stability of the algorithm, improving the convergence speed, and the simulating annealing mechanism is integrated into the internal genetic algorithm, which overcomes the shortcomings of falling into the local optimal solution. Through simulation experiments, the positioning accuracy of the ASAGA algorithm is more than $80 \%$. Compared with AG-AC and AGA algorithms, within $2 \mathrm{~m}$, the performance is improved by about $12 \%$ and $21 \%$, respectively. The convergence rate is significantly improved compared with the above algorithms, and the localization signal coverage rate is significantly improved compared with AGA algorithm.

In the future, we will further study the influence of multipath in positioning, minimize the multipath effect in combination with multi-objective optimization, properly compensate the attenuated positioning signal in positioning, and analyze the specific factors that influence the positioning accuracy caused by multipath effect.

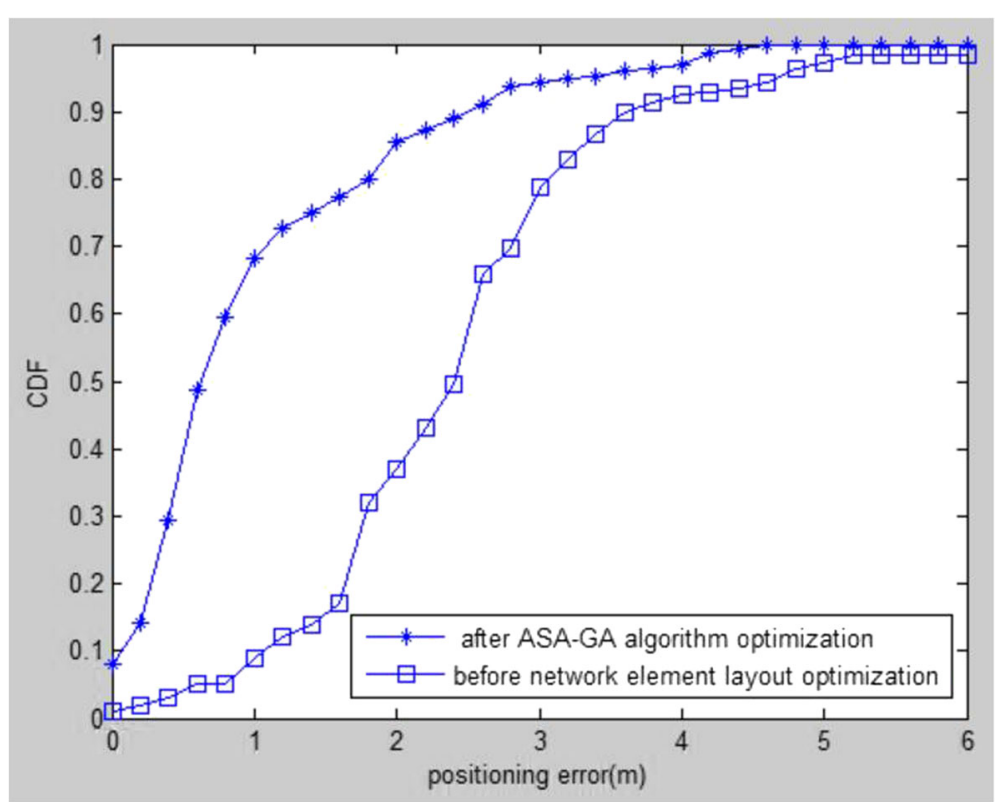

Fig. 4 Error accumulation distribution diagram before and after optimization of network elements layout. The curve with squares represents before network element layout optimization and the curve with stars represents after ASA-GA algorithm optimization 


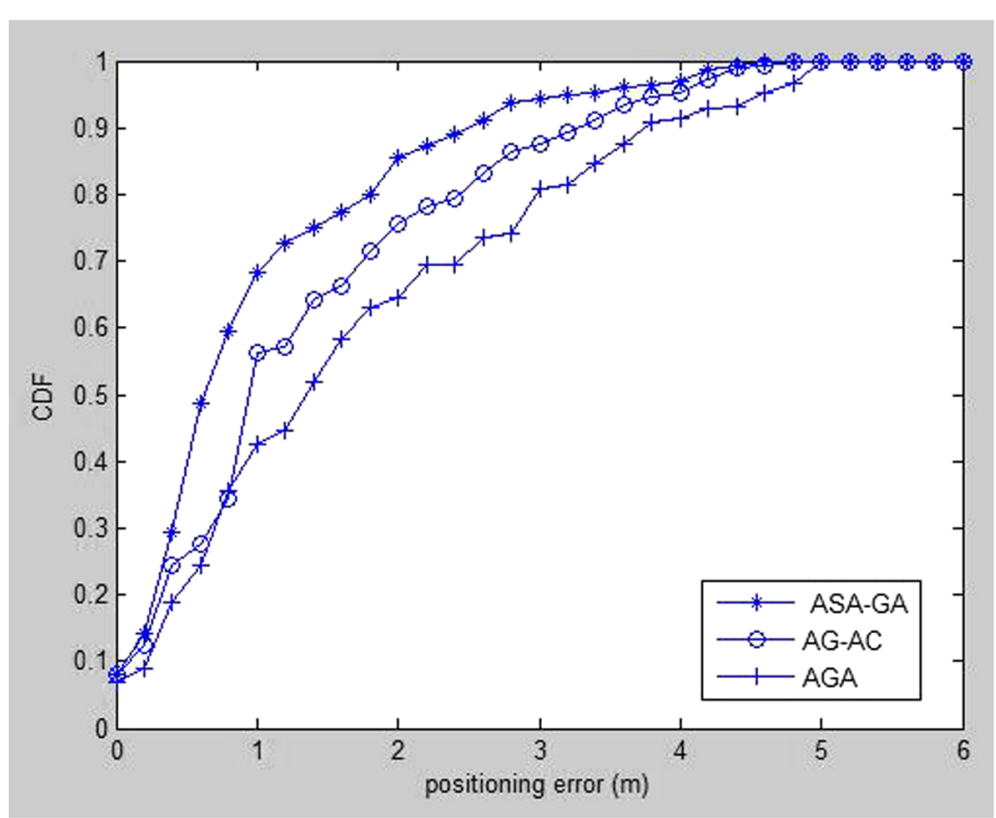

Fig. 5 Three optimization algorithms positioning error accumulation distribution diagram. The curve with circles represents after AG-AC algorithm optimization, the curve with stars represents after ASA-GA algorithm optimization, and the curve with crosses represents after AGA algorithm optimization

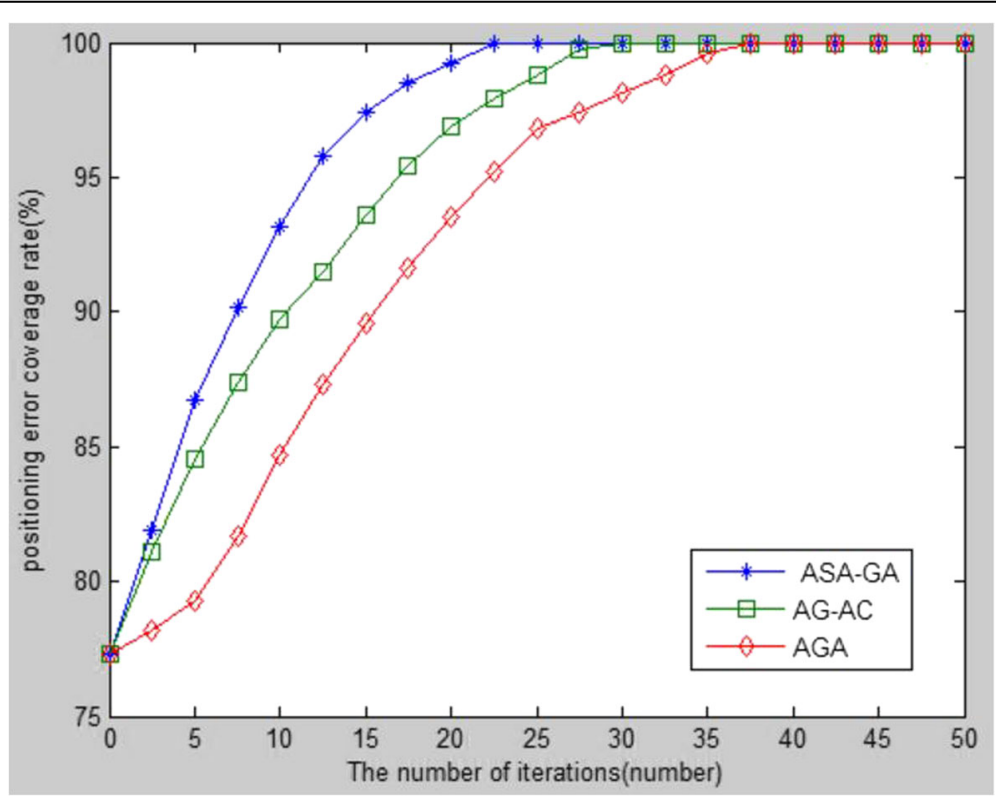

Fig. 6 Comparison of convergence of the three algorithms. The green curve with squares represents after AG-AC algorithm optimization, the curve with stars represents after ASA-GA algorithm optimization, and curve with diamonds represents after AGA algorithm optimization 


\section{Abbreviations}

NLOS: Non-line of sight; ASA-GA: Adaptive Simulated Annealing and Genetic Algorithm; AG-AC: Adaptive Genetic Combining Ant Colony; AGA: Adaptive Genetic Algorithm; GPS: Global Positioning System; EM: Electromagnetic; OAM: Orbital angular momentum; WiFi: Wireless Fidelity; UWB: Ultra wide band; 5G: Fifth generation; 3D: Three-dimensional; GDoP: Geometric Dilution of Precision; NP: Non-deterministic polynomial; PSO-ANN: Particle Swarm Optimization - Artificial Neural Network; PSO: Particle swarm optimization

\section{Authors' contributions}

XMY contributed in investigation, methodology, draft manuscript writing, manuscript reviewing, and editing. HQW contributed in the overall design and network element optimization layout model. HWL contributed in the design of models and algorithms, reviewing and editing the manuscript, and funding acquisitions. XBL contributed in software and hardware development, simulations, result analysis and reviewing and editing the manuscript. JQW contributed in reviewing and editing the manuscript. All authors read and approved the final manuscript.

\section{Funding}

This research was funded by 3 foundation items: National Natural Science Foundation of China (No. 61872104 and 61901134), the National Science and Technology Major Project of China (No. 2016ZX03001023-005), and Basic business project in education department of Heilongjiang province of China (No. 135109243)

\section{Availability of data and materials}

The datasets used and analyzed during the current study are available from the corresponding author on a reasonable request.

\section{Competing interest}

The authors declare that they have no competing interests.

\section{Author details}

${ }^{1}$ College of Computer Science and Technology, Harbin Engineering University, Harbin, Heilongjiang province, China. ${ }^{2}$ College of Computer and Control Engineering, Qiqihar University, Qiqihar, Heilongjiang province, China.

Received: 15 July 2019 Accepted: 11 November 2019 Published online: 27 December 2019

\section{References}

1. Zhou M, Zhang Q, Tian Z, et al. IMLours: Indoor mapping and localization using time-stamped WLAN received signal strength// Wireless Communications and NETWORKING Conference. IEEE, 2015:1817-1822.

2. M. Terán, J. Aranda, H. Carrillo, et al., loT-based system for indoor location using bluetooth low energy//Communications and Computing (COLCOM), 2017 IEEE Colombian Conference on. IEEE, 1-6 (2017)

3. M. Chen, L. Jiang, W. Sha, Ultrathin complementary metasurface for orbital angular momentum generation at microwave frequencies[]]. IEEE Trans Antennas Propagation 65(1), 396-400 (2017)

4. Chen M , Jiang L J , Sha W. Detection of orbital angular momentum with metasurface at microwave band. IEEE Antennas Wireless Propagation Lett, 2017:1-1.

5. X. Zeng, W. Lin, A kind of improved fingerprinting indoor location method based on WiFi//AIP Conference Proceedings. AIP Publishing 1864(1), 020052 (2017)

6. C. Yara, Y. Noriduki, S. Ioroi, et al., Design and implementation of map system for indoor navigation - an example of an application of a platform which collects and provides indoor positions// IEEE International Symposium on Inertial Sensors and Systems. IEEE, 1-4 (2015)

7. Z. Yifan, Z. Zhifeng, Towards 5G: heterogeneous cellular network architecture design based on intelligent SDN paradigm. Telecommun Sci 32(6), 28 (2016)

8. J. Wu, Q. Gang, K. Pengbin, Emerging 5 G multi-carrier chaotic sequence spread spectrum technology for underwater acoustic communication. Complexity (2018)

9. A. Bais, H. Kiwan, Y. Morgan, On optimal placement of short range base stations for indoor position estimation[J]. J. Appl. Res. Technol 12(5), 886897 (2014)
10. S. Yang, F. Dai, M. Cardei, et al., On connected multiple point coverage in wireless sensor networks[J]. Int. J. Wireless Inf. Networks 13(4), 289-301 (2006)

11. M. Hefeeda, M. Bagheri, Randomized k-coverage algorithms for dense sensor networks//INFOCOM 2007. 26th IEEE International Conference on Computer Communications. IEEE, 2376-2380 (2007)

12. Kalantari E, Yanikomeroglu H, Yongacoglu A. On the number and 3-D placement of drone base stations in wireless cellular networks/Nehicular Technology Conference (VTC-Fall), 2016 IEEE 84th. IEEE, 2016: 1-6.

13. I. Sharp et al., "Eanalysis for positioning system design," IEEE Trans. Vehicular Technol 58(7), 3371-3382 (2009)

14. N. Levanon, "Lowest GDOP in 2-D scenarios," IEE Proc. Radar Sonar Novigation 147(3), 149-155 (2000)

15. M.A. Spirito, On the accuracy of cellular mobile station location estimation. EEE Trans Vehicular Technol 50(3), 674-685 (May 2001)

16. C.-H. Chen, An arrival time prediction method for bus system. IEEE Internet Things J. 10(5), 4231-4232 (2018)

17. J.G. Andrews, A.K. Gupta, H.S. Dhillon, A primer on cellular network analysis using stochastic geometry. arXiv preprint arXiv 1604, 03183 (2016)

18. J. Zhou et al., Landmark placement for wireless localization in rectangularshaped industrial facilities. IEEE Trans Vehicular Technol 59(6), 3081-3090 (Jul 2010)

19. Y. Chen et al., in Thirrd Annual IEEE Communications Society Conference on Sensor and Ad Hoc Communications and Networks. A practical approach to landmark deployment for indoor localization (2006), pp. 365-373

20. C. Shijun, H. Wang, C. Dawei, et al., Base Station layout optimization algorithm based on improved tabu search[J]. Comp Eng Sci 40(02), 341-347 (2018)

21. H. Zhang, S. Zhang, W. Bu, A clustering routing protocol for energy balance of wireless sensor network based on simulated annealing and genetic algorithm. Int J Hybrid Inf Technol 7(2), 71-82 (2014)

22. M.B. Pereira, F.R.P. Cavalcanti, T.F. Maciel, Particle swarm optimization for base station placement//Telecommunications Symposium (ITS), 2014 International. IEEE, 1-5 (2014)

23. Meng $\mathrm{H}$, Long $\mathrm{F}$, Guo L, et al. Cooperating base station location optimization using genetic algorithm//Control and Decision Conference (CCDC), 2016 Chinese. IEEE, 2016: 4820-4824.

24. J. Munyaneza, A. Kurien, Optimization of antenna placement in $3 \mathrm{G}$ networks using genetic algorithm[J]. Commun Inf Technol 36(5), 70-80 (2009)

25. H. Wang, L. Xiubing, L. Hongwu, et al., Method of diamond supplement for indoor micro base station placement. J Beijing Univ Posts Telecommun 41(1), 51-58, 87 (2018)

26. S.K. Gharghan, R. Nordin, M. Ismail, et al., Accurate wireless sensor localization technique based on hybrid PSO-ANN algorithm for indoor and outdoor track cycling[J]. IEEE Sensors J. 16(2), 529-541 (2016)

27. Y. Gao, S.L. Xie, Particle swarm optimization algorithms based on simulated annealing. Comput Eng Appl 40, 47-50 (2004)

28. Zhang $Q$, Wang J, Jin C, et al. Localization algorithm for wireless sensor network based on genetic simulated annealing algorithm//Wireless Communications, Networking and Mobile Computing, 2008. WiCOM'08. 4th International Conference on. IEEE, 2008: 1-5.

29. C. Liu, G. Cao, Spatial-temporal coverage optimization in wireless sensor networks[J]. IEEE Trans. Mob. Comput. 10(4), 465-478 (2011)

30. Z. Hao, T. Ran, L. Zhi-yong, et al., A feature selection method based on adaptive simulated annealing genetic algorithm [J]. Acta Armam 30(1), 8185 (2009)

\section{Publisher's Note}

Springer Nature remains neutral with regard to jurisdictional claims in published maps and institutional affiliations. 